\title{
FINE AND PARABOLIC LIMITS FOR SOLUTIONS OF SECOND-ORDER LINEAR PARABOLIC EQUATIONS ON AN INFINITE SLAB
}

\author{
BY
}

\author{
B. A. MAIR
}

\begin{abstract}
This paper investigates the boundary behaviour of positive solutions of the equation $L u=0$, where $L$ is a uniformly parabolic second-order differential operator in divergence form having Hölder-continuous coefficients on $X=\mathbf{R}^{n} \times$ $(0, T)$, where $0<T<\infty$. In particular, the notion of semithinness for the potential theory on $X$ associated with $L$ is introduced, and the relationships between fine, semifine and parabolic convergence at points of $\mathbf{R}^{n} \times\{0\}$ are studied.

The abstract Fatou-Naim-Doob theorem is used to deduce that every positive solution of $L u=0$ on $X$ has parabolic limits Lebesgue-almost-everywhere on $\mathbf{R}^{n} \times\{0\}$. Furthermore, a Carleson-type result is obtained for solutions defined on a union of parabolic regions.
\end{abstract}

0. Introduction. Let $L$ be a second-order linear parabolic differential operator having divergence structure on $X=\mathbf{R}^{n} \times(0, T)$ where $0<T<\infty$. The coefficients of $L$ are assumed to be such that the classical fundamental solution exists, the results in [2] for classical solutions hold, and the solutions of $L u=0$ form a strong harmonic space in the sense of Bauer [3].

In the particular case of the heat operator $\Delta_{x}-\partial / \partial t$, Doob [11] proved the almost everywhere convergence through parabolic regions of quotients of positive solutions on $X$. Hattemer [16] showed that if $E \subset \mathbf{R}^{n}, u$ is a solution of the heat equation on $X$, and for each $b \in E, u$ is bounded on a parabolic region with vertex $b$, then $u$ has finite parabolic limits almost everywhere on $E$ (cf. [7]). Results of Kemper [20] imply a Carleson-type result for solutions of the heat equation (i.e. Hattemer's result holds if $u$ is only upper or lower bounded on each parabolic region).

For certain parabolic operators with divergence structure, Johnson [19] proved the Lebesgue-almost-everywhere convergence through parabolic regions of positive solutions on $X$.

In this paper, Johnson's result for $L$ is deduced from the abstract Fatou-NaimDoob theorem. Also, a Carleson-type result is established for solutions of $L u=0$ defined on a union of parabolic regions. The methods employed in this paper were inspired mainly by those in [6, 21 and 22].

Received by the editors February 16, 1983 and, in revised form, July 6, 1983.

1980 Mathematics Subject Classification. Primary 31B25; Secondary 35K20, 35K05.

Key words and phrases. Harmonic, fine limit, semifine limit, parabolic limit, local Fatou theorem.

'This research is part of the author's doctoral thesis done at McGill University, Montreal. 
Basically this paper shows that all the ideas introduced by Brelot and Doob in [6] for the study of nontangential convergence for quotients of positive solutions of Laplace's equation can be used to study parabolic convergence for positive solutions of certain parabolic equations. In particular, semithinness, which was not used in [22], is seen to be also useful in the parabolic case. As in the case of Laplace's equation, to prove the local theorem it is necessary to obtain a suitable relationship between the ideal boundaries of $X$ and certain subsets of $X$ (cf. [21]). This is done in $\$ 7$ by using the integral representation theorem in [18] and on adaptation of the methods used in the appendix of [21].

I would like to thank Professor J. C. Taylor of McGill University for suggesting this problem and the stimulating discussions we had.

1. Preliminaries. Throughout this paper $0<T<\infty$ and $n \in \mathbf{N}$ are fixed and $X$ denotes the infinite slab

$$
\mathbf{R}^{n} \times(0, T)=\left\{(x, t):\left(x_{1}, x_{2}, \ldots, x_{n}\right) \in \mathbf{R}^{n}, 0<t<T\right\} .
$$

The lower boundary $\mathbf{R}^{n} \times\{0\}$ will be identified with $\mathbf{R}^{n}$. The linear second-order uniformly parabolic differential operator $L$ with divergence structure is defined by

$$
L u=\sum_{i, j=1}^{n} \frac{\partial}{\partial x_{j}}\left(a_{i j}(x, t) \frac{\partial u}{\partial x_{i}}\right)+\sum_{j=1}^{n} b_{j}(x, t) \frac{\partial u}{\partial x_{j}}+c(x, t) u-\frac{\partial u}{\partial t},
$$

where the coefficients are bounded, Hölder-continuous real-valued functions on $\mathbf{R}^{n} \times[0, T] ; \partial a_{i j} / \partial x_{k}$ exists and is Hölder-continuous for any $i, j, k \in\{1,2, \ldots, n\}$; $\left(a_{i j}\right)$ is a symmetric matrix and there exists a constant $M>0$ such that

$$
\sum_{i, j=1}^{n} a_{i j}(x, t) y_{i} y_{j} \geqslant M^{-1}\|y\|^{2}, \quad\left|a_{i j}(x, t)\right| \leqslant M
$$

for all $i, j \in\{1,2, \ldots, n\},(x, t) \in \mathbf{R}^{n} \times[0, T]$ and $y \in \mathbf{R}^{n}$.

For any open $U \subset X$, let $\mathcal{H}(U)$ be the set of real-valued functions $u$ which are of class $\complement^{2}$ with respect to $x$, of class $\complement^{1}$ with respect to $t$, and satisfy $L u=0$ on $U$.

The following properties hold.

(P1) $(X, \mathcal{K})$ is a strong harmonic space in the sense of [3] (cf. [15, 3, 10, Exercise 3.3.5]). A function in $\mathcal{H}(U)$ is said to be harmonic on $U$.

(P2) The classical fundamental solution $\Gamma$ for the operator $L$ exists and there are positive constants $P, p_{1}, p_{2}$ such that

$$
P^{-1} W_{1} \leqslant \Gamma \leqslant P W_{2} \text { on } X \times X
$$

where $W_{i}$ is the fundamental solution for the operator $p_{i} \Delta_{x}-\partial / \partial t$ for $i=1,2$. That is,

$$
W_{i}(x, t ; y, s)=\left\{\begin{array}{l}
{\left[4 p_{i} \pi(t-s)\right]^{-n / 2} \exp \left[-\frac{\|x-y\|^{2}}{4 p_{i}(t-s)}\right], \quad \text { if } t>s,} \\
0, \quad \text { if } t \leqslant s .
\end{array}\right.
$$

(cf. [2, Theorem 7]). For each $b \in \mathbf{R}^{n}$, let $K_{b}(x, t)=\Gamma(x, t ; b, 0)$ for all $(x, t) \in X$. 
(P3) For each nonnegative harmonic function $u$ on $X$ there exists a unique Borel measure $\mu$ on $\mathbf{R}^{n}$ such that

$$
u(x, t)=\int K_{b}(x, t) d \mu(b) \quad \text { for all }(x, t) \in X .
$$

$\mu$ is called the representing measure for $u$. Conversely, if $\mu$ is a Borel measure on $\mathbf{R}^{n}$ such that $u(x, t)=\int K_{b}(x, t) d \mu(b)$ is finite for all $(x, t) \in X$, then $u$ is a nonnegative harmonic function on $X$ (cf. [2, Theorem 12 and Corollary 12.1]).

(P4) For each $b \in \mathbf{R}^{n}, K_{b}$ is a minimal harmonic function on $X$ (cf. [2, Corollary 12.2]).

(P5) $\int K_{b}(x, t) d b=1$ for all $(x, t) \in X$ (cf. [1]). That is, Lebesgue $n$-dimensional measure represents 1 .

These properties, together with basic results in axiomatic potential theory (cf. $[3,10])$ imply that hypotheses (1)-(11) in [26] are satisfied. The following result is therefore a consequence of the Fatou-Naim-Doob theorem (cf. [26]).

THEOREM 1.1. Let $u, v$ be positive harmonic functions on $X$ represented by measures $\mu, \nu$, respectively. Then $u / v$ has fine limit $d \mu / d \nu \nu$-a.e. on $\mathbf{R}^{n}$.

For the reader's convenience, the concept of fine limit is now defined.

DEFINITION 1.2 (CF. [13, 26]). (i) For any nonnegative superharmonic function $u$ on $X$ and $E \subset X$, the reduced function of $u$ on $E$ is defined on $X$ by

$R_{E} u(x, t)=\inf \{w(x, t): w$ is nonnegative, superharmonic on $X$ and $w \geqslant u$ on $E\}$. $\hat{R}_{E} u$ denotes its lower semicontinuous regularisation and is called the balayage of $u$ on $E$.

(ii) A set $E \subset X$ is said to be thin at $b \in \mathbf{R}^{n}$ if $R_{E} K_{b} \neq K_{b}$ or, equivalently, $\hat{R}_{E} K_{b} \neq K_{b}$.

(iii) The fine filter at $b, \mathscr{F}(b)=\{E \subset X: X \backslash E$ is thin at $b\}$. Limits along this filter are called fine limits.

Throughout this paper, $C$ denotes a general positive constant (not necessarily the same at different occurrences) which may depend on $n, P, p_{1}, p_{2}$ and other constants.

REMARK 1.3. The function $c$ is bounded, hence there exists a constant $q>0$ such that $c \leqslant q$. Then, for sufficiently differentiable $u, L\left(e^{q t} u\right)=e^{q t}(L u-q u)$. Hence $L\left(t e^{q t}\right)<0$.

Now, in the case of the potential theory associated to the Laplacian, the complement of a Martin neighbourhood of a point on the Martin boundary is thin at that point. The following lemma establishes a similar result for parabolic potential theory on $X$.

LEMMA 1.4. For any $b \in \mathbf{R}^{n}$ and neighbourhood $U$ of $b$ in $\mathbf{R}^{n+1}$, the set $X \backslash U$ is thin at $b$.

Proof. Let $b$ and $U$ be as in the hypothesis. Then there exists $\delta>0$ such that $\{(x, t):\|x-b\| \leqslant \delta, 0<t \leqslant \delta\} \subset U$. Since $\Gamma \leqslant P W_{2}$ and $e^{-\lambda} \leqslant\left(2^{-m} m !\right)^{1 / 2} \lambda^{-m / 2}$ 
for $m=0, n+2$, it follows that

$$
K_{b}(x, t) \leqslant C t \leqslant C t e^{q t} \text { for all }(x, t) \in X \backslash U .
$$

Then, by Remark 1.3, $R_{X \backslash U} K_{b}(x, t) \leqslant C t e^{q t}$ for all $(x, t) \in X$. Hence $R_{X \backslash U} K_{b}(x, t)$ $\rightarrow 0$ as $(x, t) \rightarrow b$, so $R_{X \backslash U} K_{b} \neq K_{b}$.

A useful characterisation of thinness is provided by the following result (cf. [23, Théorème 4 ; 17, Lemma (5.1)]).

Proposition 1.5. Let $E \subset X$ and $b \in \mathbf{R}^{n}$. If $E$ is thin at $b$ then for any sequence $\left\{U_{m}\right\}$ of neighbourhoods of $b$ in $\mathbf{R}^{n+1}$ decreasing to $\{b\}$ and $(x, t) \in X$,

$$
\lim _{m \rightarrow \infty} \hat{R}_{E(m)} K_{b}(x, t)=0 \text {, }
$$

where $E(m)=E \cap U_{m}$.

Conversely, if there exist $(x, t) \in X$ and a sequence $\left\{U_{m}\right\}$ as above such that $\lim _{m \rightarrow \infty} \hat{R}_{E(m)} K_{b}(x, t)=0$, then $E$ is thin at $b$.

Proof. Assume $E$ is thin at $b$. Define

$$
u(x, t)=\lim _{m \rightarrow \infty} \hat{R}_{E(m)} K_{b}(x, t) \quad \text { for each }(x, t) \in X .
$$

Then $u$ is nonnegative and harmonic on $X$ because $\hat{R}_{E(m)} K_{b}$ is harmonic on $X \backslash U_{m}$ and the Doob convergence property for a decreasing sequence of harmonic functions can be applied. However, $\hat{R}_{E(m)} K_{b}$ is a potential for each $m$, hence $u=0$.

Conversely, if $\lim _{m \rightarrow \infty} \hat{R}_{E(m)} K_{b}(x, t)=0$ for some $(x, t) \in X$, then there exists $m$ such that $\hat{R}_{E(m)} K_{b}(x, t)<K_{b}(x, t)$, so $E(m)$ is thin at $b$. Hence $E$ is thin at $b$ by Lemma 1.4.

The solution of the Dirichlet problem in the sense of Perron-Wiener-Brelot (cf. $[10$, p. 18]) plays a major role in examining the relationships between fine, semifine and parabolic limits. Results in [2] show that $K_{b} d b$ is the harmonic measure on $X$.

2. Semifine and parabolic limits for arbitrary functions. In [6] Brelot and Doob defined "semi-effilement" at a point of $\mathbf{R}^{n}$ for classical potential theory on $X$. In order to define semithinness at a point of $\mathbf{R}^{n}$ for parabolic potential theory, it is necessary to modify their definition so as to take account of the "parabolic scaling" of the heat equation.

In the remainder of this paper, $\gamma$ denotes a fixed number and $0<\gamma<1$.

Definition 2.1. (i) For any $b \in \mathbf{R}^{n}$ and $m \in \mathbf{N}$, define

$$
R_{m}(b)=\left\{(x, t) \in X:\|x-b\|<\gamma^{m}, t<\gamma^{2 m}\right\},
$$

and

$$
J_{m}(b)=R_{m}(b) \backslash R_{m+1}(b) .
$$

$J_{m}(b)$ will be denoted by $J_{m}$ when the context determines $b$.

(ii) A set $E \subset X$ is said to be semithin at $b \in \mathbf{R}^{n}$ if there exists $(x, t) \in X$ such that $\lim _{m \rightarrow \infty} \hat{R}_{E \cap J_{m}} K_{b}(x, t)=0$.

(iii) For each $b \in \mathbf{R}^{n}, \delta(b)=\{E \subset X: X \backslash E$ is semithin at $b\}$ is called the semifine filter at $b$. For any function $f$, semifine $\lim f(b)$ denotes the limit of $f$ along $\delta(b)$. Similarly, semifine $\lim \sup f(b)$ denotes the limsup of $f$ along $\delta(b)$ and semifine liminf $f(b)$ denotes the lim inf of $f$ along $\delta(b)$. 
REMARK 2.2. (i) For any $(x, t)$ and $\left(x_{0}, t_{0}\right) \in X$, there exists $\alpha \in \mathbf{R}$ such that $u(x, t) \leqslant \alpha u\left(x_{0}, t_{0}\right)$ for all positive harmonic functions $u$ on $X$ (cf. [2, Theorem $\left.\mathrm{H}\right]$ ). Hence, $E \subset X$ is semithin at $b \in \mathbf{R}^{n}$ iff there exists $t_{0} \in(0, T)$ such that $\lim _{n \rightarrow \infty} \hat{R}_{E \cap J_{m}} K_{b}(x, t)=0$ for all $t \in\left(0, t_{0}\right)$.

(ii) It is clear from Proposition 1.5 that $\mathscr{F}(b) \subset \mathcal{S}(b)$ for all $b \in \mathbf{R}^{n}$,

Definition 2.3. (i) For any $\alpha>0$ and $b \in \mathbf{R}^{n}$, the region

$$
P(b ; \alpha)=\left\{(x, t) \in X:\|x-b\|^{2}<\alpha t\right\}
$$

is called the parabolic region with aperature $\alpha$ and vertex $b$. For any $\delta>0, P(b ; \alpha, \delta)$ denotes $P(b ; \alpha) \cap\{(x, t) \in X: t<\delta\}$.

(ii) A real-valued function $f$ is said to have parabolic limit $\lambda$ at $b \in \mathbf{R}^{n}$ if $\left\{f\left(x_{n}, t_{n}\right)\right\}$ converges to $\lambda$ for every sequence $\left\{\left(x_{n}, t_{n}\right)\right\}$ which converges to $b$ within a parabolic region.

(iii) $\mathcal{P}(b)=\{E \subset X$ : for each $\alpha>0$ there exists $\delta>0$ such that $\mathscr{P}(b ; \alpha, \delta) \subset E\}$ is called the parabolic filter at $b$.

It is clear that parabolic convergence at $b$ is equivalent to convergence along $\mathscr{P}(b)$.

Proposition 2.4 (CF. [6, ThEorème 1]). For each $b \in \mathbf{R}^{n}, \mathcal{P}(b) \subset \delta(b)$. Hence, any function having parabolic limit at $b$ has the same semifine limit at $b$.

Proof. For each $m \in \mathbf{N}$, let

$$
\begin{gathered}
B_{m}=\left\{y \in \mathbf{R}^{n}: \gamma^{m+2} \leqslant\|y-b\|<\gamma^{m-1}\right\}, \\
v_{m}(x, t)=\int_{B_{m}} K_{y}(x, t) d y \text { and } u_{m}(x, t)=\int_{B_{m}} W_{1}(x, t ; y, 0) d y
\end{gathered}
$$

for all $(x, t) \in X$. Then, by using the fact that $P^{-1} W_{1} \leqslant \Gamma$ and the transformations

$$
\begin{gathered}
y \in B_{m} \rightarrow \gamma^{-(m-1)}(y-b)+b \in B_{1}, \\
(x, t) \in J_{m} \rightarrow\left(\gamma^{-(m-1)}(x-b)+b, \gamma^{-2(m-1)} t\right) \in J_{1},
\end{gathered}
$$

it is clear that

$$
\begin{aligned}
\inf \left\{v_{m}(x, t):(x, t) \in J_{m}\right\} & \geqslant C \inf \left\{u_{m}(x, t):(x, t) \in J_{m}\right\} \\
& =C \inf \left\{u_{1}(x, t):(x, t) \in J_{1}\right\}>0
\end{aligned}
$$

for all $m$. Now, Let $E \in \mathscr{P}(b), F=X \backslash E$ and $0<\varepsilon<1$. Then there exists $m_{0} \in \mathbf{N}$ such that $m \geqslant m_{0}$ and $(x, t) \in F \cap J_{m}$ implies $t<\varepsilon\|x-b\|^{2}$. Since $e^{-\lambda} \leqslant n ! \lambda^{-n}$ for all $\lambda>0$, it follows that

$$
K_{b}(x, t) \leqslant C t^{n / 2}\|x-b\|^{-2 n} \quad \text { for all }(x, t) \in X .
$$

Now, $(x, t) \in J_{m}$ implies

which implies

$$
\gamma^{2(m+1)} \leqslant t<\gamma^{2 m} \text { or } \quad \gamma^{m+1} \leqslant\|x-b\|<\gamma^{m},
$$

$$
\begin{aligned}
& K_{b}(x, t) \leqslant C\left\{t\|x-b\|^{-2}\right\}^{n} \gamma^{-n(m+1)} \text { or } \\
& K_{h}(x, t) \leqslant C\left\{t\|x-b\|^{-2}\right\}^{n / 2} \gamma^{-n(m+1)} .
\end{aligned}
$$


Therefore, for $m \geqslant m_{0}$ and $(x, t) \in F \cap J_{m}$,

$$
K_{b}(x, t) \leqslant C \gamma^{-n(m+1)} \varepsilon^{n / 2} \leqslant C \gamma^{-n(m+1)} \varepsilon^{n / 2} v_{m}(x, t) .
$$

Now,

$$
v_{m}(x, t) \leqslant C t^{-n / 2}\left(\text { volume of } B_{m}\right)=C t^{-n / 2} \gamma^{n(m-1)}\left(1-\gamma^{3 n}\right) .
$$

Hence, for $m \geqslant m_{0}$ and $(x, t) \in X$,

$$
\hat{R}_{F \cap J_{m}} K_{b}(x, t) \leqslant C \gamma^{-2 n}\left(1-\gamma^{3 n}\right) t^{-n / 2} \varepsilon^{n / 2} .
$$

It follows that $F$ is semithin at $b$.

3. Nonsemithin sets. For classical potential theory on $x$, it is known (cf. [6, Théorème 3]) that if $\left\{\left(y_{m}, t_{m}\right)\right\}$ converges to $b$ within a cone, then for any $\alpha>0$ the so-called "bubble" set,

$$
\bigcup_{m=1}^{\infty}\left\{(x, t):\left\|(x, t)-\left(y_{m}, t_{m}\right)\right\|<\alpha t_{m}\right\}
$$

is not semithin at $b$.

In the case of the potential theory for the heat equation on $X$, Koranyi and Taylor [22, Proposition 4.1] proved that if $t_{m} \downarrow 0$, then for any $\alpha>0$, the set $\cup_{m=1}^{\infty}\left\{\left(x, t_{m}\right)\right.$ : $\left.\|x-b\|^{2} \leqslant \alpha t_{m}\right\}$ is not thin at $b$. This is a special case of the following result.

Proposition 3.1. Let $\left\{\left(y_{m}, t_{m}\right)\right\}$ be a sequence in $P(b ; \alpha)$ which converges to $b \in \mathbf{R}^{n}$ and let $\beta>0$. Then $\cup_{m=1}^{\infty}\left\{\left(x, t_{m}\right):\left\|x-y_{m}\right\|^{2} \leqslant \beta t_{m}\right\}$ is not semithin at $b$.

Proof. For each $m \in \mathbf{N}$, let $E_{m}=\left\{\left(x, t_{m}\right):\left\|x-y_{m}\right\|^{2} \leqslant \beta t_{m}\right\}$ and $E=\bigcup_{m=1}^{\infty} E_{m}$. Fix $\left(x_{0}, t_{0}\right) \in X$ and $m_{0} \in \mathbf{N}$ such that $t_{m}<t_{0}$ for all $m \geqslant m_{0}$. Then, $\hat{R}_{E_{m}} 1$ dominates the solution of the Dirichlet problem on the half-space $\left\{(x, t): t>t_{m}\right\}$ corresponding to the characteristic function of $E_{m}$. Therefore, by using $P^{-1} W_{1} \leqslant \Gamma$ and Proposition 1.6, it follows that for $m \geqslant m_{0}$,

$$
\hat{R}_{E_{m}} 1\left(x_{0}, t_{0}\right) \geqslant C\left(t_{0}-t_{m}\right)^{-n / 2} \exp \left\{-\frac{\left(\left\|x_{0}-y_{m}\right\|+\sqrt{\beta t_{m}}\right)^{2}}{4 p_{1}\left(t_{0}-t_{m}\right)}\right\}\left(\beta t_{m}\right)^{n / 2} .
$$

Hence,

$$
\liminf _{m \rightarrow \infty} t_{m}^{-n / 2} \hat{R}_{E_{m}} 1\left(x_{0}, t_{0}\right)>0 .
$$

Since $\|x-b\|^{2} \leqslant 2\left(\left\|x-y_{m}\right\|^{2}+\left\|y_{m}-b\right\|^{2}\right)$, it is clear that

$$
\hat{R}_{E_{m}} K_{b}\left(x_{0}, t_{0}\right) \geqslant C t_{m}^{-n / 2} \hat{R}_{E_{m}} 1\left(x_{0}, t_{0}\right) \text {. }
$$

Hence there is a constant $\delta>0$ such that $\hat{R}_{E_{m}} K_{b}\left(x_{0}, t_{0}\right) \geqslant \delta$ for all sufficiently large $m$.

Now, for each $m \geqslant m_{0}$, put $\tau_{m}=\left\{k \in \mathbf{N}: E_{m} \cap J_{k} \neq \varnothing\right\} . E \subset P(b ; \lambda)$ where $\lambda=\max \{2(\alpha+\beta), 1\}$. Hence $k \in \tau_{m}$ implies $\lambda^{-1} \gamma^{2(k+1)} \leqslant t_{m} \leqslant \gamma^{2 k}$, which implies $\tau_{m}$ is contained within an interval of fixed length $1-(\log \lambda) /(2 \log \gamma)$. Therefore, 
there exists $q \in \mathbf{N}$, independent of $m$, such that the cardinality of $\tau_{m} \leqslant q$. Now, for all sufficiently large $m$,

$$
E_{m}=\bigcup_{k \in \tau_{m}} E_{m} \cap J_{k} \quad \text { so } \quad \hat{R}_{E_{m}} K_{b} \leqslant \sum_{k \in \tau_{m}} \hat{R}_{E_{m} \cap J_{k}} K_{b} .
$$

Hence, for all sufficiently large $m$, there exists $k(m)$ such that $\hat{R}_{E \cap J_{k(m)}} K_{b}\left(x_{0}, t_{0}\right) \geqslant$ $\delta / q$. This proves $E$ is not semithin at $b$.

REMARK 3.2. It is interesting to note that, by using a similar argument, it can be shown that if $\left\{\left(y_{m}, t_{m}\right)\right\}$ converges to $b$ within a cone, then $\cup_{m=1}^{\infty}\left\{\left(x, t_{m}\right):\left\|x-y_{m}\right\|\right.$ $\left.\leqslant \beta t_{m}\right\}$ is not semithin (in the classical sense) at $b$. This exhibits a smaller nonsemithin set than the standard "bubble" set.

4. Semifine and parabolic limits for harmonic functions. In [22, Theorem 4.2], Koranyi and Taylor used the fine limit theorem and a reduction theorem [22, Theorem 1.2] to deduce Doob's result (cf. [11]) that every positive solution of the heat equation on $X$ has finite parabolic limits a.e. on $\mathbf{R}^{n}$. The following theorem uses their method to obtain the same result for the linear second-order parabolic differential operators $L$ introduced in $§ 1$, thus deriving Johnson's result (cf. [19]) from the theory of fine convergence.

THEOREM 4.1. Let $b \in \mathbf{R}^{n}$ and $u>0$ be harmonic on $P(b ; \beta, \delta)$ for some $\beta, \delta>0$. Then, for any $\alpha<\beta$, u has limit 0 along $\delta(b)$ restricted to $P(b ; \alpha)$ implies $u(x, t) \rightarrow 0$ as $(x, t) \rightarrow b$ within $P(b ; \alpha)$.

Consequently, if $u>0$ and satisfies $L u=0$ on $X$, then $u$ has parabolic limit $d \mu / d b$ for a.e. $b \in \mathbf{R}^{n}$, where $\mu$ is the representing measure for $u$.

Proof. Suppose there exists a sequence $\left\{\left(y_{m}, t_{m}\right)\right\}$ converging to $b$ such that $\left\|y_{m}-b\right\|^{2}<\alpha t_{m}$, and a constant $\lambda>0$ such that $u\left(y_{m}, t_{m}\right) \geqslant \lambda$ for all $m \in \mathbf{N}$. Let $\eta$ be such that $\alpha<\eta<\beta$. Then by putting $\Omega=\left\{x \in \mathbf{R}^{n}:\|x-b\|^{2}<\eta t\right\}, \Omega^{\prime}=\{x \in$ $\left.\mathbf{R}^{n}:\|x-b\|^{2}<\alpha t\right\}$ in the Harnack-type inequality of Aronson and Serrin (cf. [2, Theorem $\mathrm{H}])$, it follows that there is a constant $\lambda_{1}>0$ such that $u(x, 2 t) \geqslant \lambda_{1} u(y, t)$ for all $0<t<\min (1, \delta / 2),\|x-b\|^{2}<\alpha t,\|y-b\|^{2}<\alpha t$. Hence, $u \geqslant \lambda_{1} \lambda$ on $E=\cup_{m=1}^{\infty}\left\{\left(x, 2 t_{m}\right):\|x-b\|^{2} \leqslant \alpha t_{m}\right\}$, which is not semithin at $b$. Hence $u$ does not have semifine limit 0 at $b$.

Consequently, for any $b \in \mathbf{R}^{n}, u$ has fine limit 0 at $b$ implies $u$ has parabolic limit 0 at $b$. The theorem is completed by using Theorem 1.2 in [22] (cf. §8).

5. Parabolic and fine limits almost everywhere. In [6] it was proved that if $f$ : $X \rightarrow \mathbf{R}$ and $E \subset \mathbf{R}^{n}$ is the set of points at which $f$ has a finite nontangential limit, then $f$ converges finely a.e. on $E$. However, the method used there is rather involved. The simpler method developed by Hunt and Wheeden (cf. [17, Theorem (5.7)]) will be used here to prove an analogous result.

Lemma 5.1 (CF. [6, ThEorème 8]). Let $A \subset \mathbf{R}^{n}$ and $W \subset X$ be such that for each $b \in A, W$ contains a truncated parabolic region with vertex $b$. Then $X \backslash W$ is thin at a.e. $b \in A$. 
Proof. By considering parabolic regions of rational aperature and height, it suffices to consider the case when, for each $b \in A, W$ contains the region $P(b ; \alpha, \delta)$ with $\alpha, \delta$ fixed. Furthermore, in this case, $P(b ; \alpha, \delta)$ is contained in $W$ for every adherent point $b$ of $A$. Hence $A$ can be assumed to be closed.

Let $v$ be the solution of the Dirichlet problem on $X$ corresponding to the characteristic function of $A$. Then, by the Fatou-Naim-Doob theorem, $v$ has fine limit 1 a.e. on $A$. Therefore, for any $0<\lambda<1$, the set $E_{\lambda}=\{(x, t) \in X: v(x, t) \leqslant \lambda\}$ is thin at a.e. $b \in A$. Now, suppose $(x, t) \in X \backslash W$ and $t<\delta$. Let $D=\left\{b \in \mathbf{R}^{n}\right.$ : $\left.\|x-b\|^{2}<\alpha t\right\}$. Then

$$
\begin{aligned}
v(x, t) & \leqslant 1-C t^{-n / 2} \int_{D} \exp \left(-\frac{\|x-b\|^{2}}{4 p_{1} t}\right) d b \\
& \leqslant 1-C t^{-n / 2} e^{-\alpha / 4 p_{1}}(\alpha t)^{n / 2}=1-C<1 .
\end{aligned}
$$

Hence, $X \backslash W \subset E_{\lambda} \cup\{(x, t) \in X: t \geqslant \delta\}$ for some choice of $\lambda$.

The main result in this section will now be stated.

THEOREM 5.2. Let $f$ be a real-valued function on $X$ and $A \subset \mathbf{R}^{n}$ the set of points at which $f$ has a parabolic limit $\psi(b)$ at $b \in A$. Then $f$ converges finely to $\psi$ a.e. on $A$.

Proof. Fix an $\alpha>0$. Then for each $b \in A$ and $m \in \mathbf{N}$ there exists a positive rational number $\delta_{m}(b)$ such that $|f(x, t)-\psi(b)|<1 / m$ for all $(x, t) \in$ $P\left(b ; \alpha, \delta_{m}(b)\right)$. By dividing $A$ into a countable number of subsets, one can assume $\delta_{m}(b)=\delta_{m}$ for all $b \in A$. For $b \in A, m \in \mathbf{N}$, define

$$
Q_{m}(b)=\bigcup\left\{P\left(y ; \alpha, \delta_{m}\right): y \in A, P\left(b ; \alpha, \delta_{m}\right) \cap P\left(y ; \alpha, \delta_{m}\right) \neq \varnothing\right\} .
$$

Then

$$
Q_{m}(b)=\bigcup\left\{P\left(y ; \alpha, \delta_{m}\right): y \in A,\|y-b\|^{2} \leqslant 4 \alpha \delta_{m}\right\},
$$

which contains $W_{m} \cap\left\{(x, t) \in X:\|x-b\|^{2} \leqslant \alpha \delta_{m}, t<\delta_{m}\right\}$, where $W_{m}=$ $\cup_{y \in A} P\left(y ; \alpha, \delta_{m}\right)$. Hence $X \backslash Q_{m}(b)$ is thin at $b$ if $X \backslash W_{m}$ is thin at $b$. Now, by Lemma 5.1, for each $m$ there exists $D_{m} \subset A$ having zero Lebesgue measure such that for all $m \in \mathbf{N}, X \backslash W_{m}$ is thin at each $b \in A \backslash D_{m}$. Therefore, for all $m \in \mathbf{N}$, $X \backslash Q_{m}(b)$ is thin at each $b \in A \backslash \cup_{m=1}^{\infty} D_{m}$ and from (i), $Q_{m}(b) \subset\{(x, t) \in X$ : $|f(x, t)-\psi(b)|<3 / m\}$. The result follows.

REMARK 5.3. It is clear from the proof of the above theorem that $f$ converges finely to $\psi$ a.e. on $A$ if at each $b \in A, f$ has parabolic limit $\psi(b)$ relative to some parabolic region $P(b ; \alpha(b))$.

This remark corresponds to that made by Brelot and Doob for the case of the Laplacian (cf. [6, p. 412, footnote (11)]).

6. Semifine and parabolic limits for solutions of the heat equation. In case $L$ is the heat operator, this section improves the first part of Theorem 4.1 by using a stronger Harnack-type inequality.

In [6, p. 401], it is stated that the Harnack constant $\theta(\rho), 0<\rho<1$, for which $u(x) / u(y) \leqslant \theta(\rho)$ for all $\|x-y\| \leqslant \rho \alpha$ and for all positive solutions $u$ of Laplace's equation on $\left\{x \in \mathbf{R}^{n}:\|x-y\|<\alpha\right\}$, has the property that $\lim _{\rho \rightarrow 1} \theta(\rho)=1$. This 
plays an important role in [6, Théorème 3 and 17, Theorem 5.5]. The next result demonstrates a Harnack-type inequality for positive solutions of the heat equation $\Delta_{x} u-u_{t}=0$ on $X$ in which the Harnack constant possesses an analogous property. As this result is proved only for solutions defined on all of $X$, the local nature of Theorème 3 in [6] is lost in this case. In view of this, it is interesting to ask if the Harnack constant for solutions defined only on a finite rectangular domain possesses a similar property. This is an open question.

Proposition 6.1. (i) For each $\rho>1$ there exists $\theta(\rho)$ such that for all positive solutions $u$ of the heat equation on $X$,

$$
u(x, \rho t) \geqslant \theta(\rho) u(y, t) \quad \text { if }\|x-y\|^{2} \leqslant(\rho-1)^{2} t .
$$

Further, $\lim _{\rho \rightarrow 1} \theta(\rho)=1$.

(ii) For each $0<\rho<1$, there exists $\phi(\rho)$ such that for all positive solutions $u$ of the heat equation on $X$,

$$
u(x, \rho t) \leqslant \phi(\rho) u(y, t) \quad \text { if }\|x-y\|^{2} \leqslant \rho^{-1}(1-\rho)^{2} t .
$$

Further, $\lim _{\rho \rightarrow 1} \phi(\rho)=1$.

Proof. For the heat equation,

$$
K_{b}(x, t)=(4 \pi t)^{-n / 2} \exp \left(-\|x-b\|^{2} / 4 t\right)
$$

for all $(x, t) \in X$.

$$
\frac{K_{b}(x, \rho t)}{K_{b}(y, t)}=\rho^{-n / 2} \exp \left\{\frac{\|y-b\|^{2}}{4 t}-\frac{\|x-b\|^{2}}{4 \rho t}\right\} .
$$

The triangle inequality implies

$$
\rho(\rho-1)\|y-b\|^{2}+\rho\|x-y\|^{2} \geqslant(\rho-1)\|x-b\|^{2},
$$

which implies

Hence,

$$
\|y-b\|^{2}-\rho^{-1}\|x-b\|^{2} \geqslant-(\rho-1)^{-1}\|x-y\|^{2} .
$$

$$
\begin{aligned}
K_{b}(x, \rho t) & \geqslant \rho^{-n / 2} \exp \left\{-\|x-y\|^{2} / 4(\rho-1) t\right\} K_{b}(y, t) \\
& \geqslant \rho^{-n / 2} \exp ((1-\rho) / 4) K_{b}(y, t), \quad \text { if }\|x-y\|^{2} \leqslant(\rho-1)^{2} t .
\end{aligned}
$$

Set $\theta(\rho)=\rho^{-n / 2} \exp ((1-\rho) / 4)$ and $\phi(\rho)=\left\{\theta\left(\rho^{-1}\right)\right\}^{-1}$.

In the case of $n=1$, this result can be deduced from Theorem 1 in [25].

THEOREM 6.2 (CF. [6, THEOREME 6]). Let u be a positive solution of the heat equation on $X$ having $\lambda$ as a parabolic cluster value at $b \in \mathbf{R}^{n}$. Then

$$
\text { semifine } \liminf u(b) \leqslant \lambda \leqslant \text { semifine } \lim \sup u(b) .
$$

Consequently, for any $b \in \mathbf{R}^{n}$,

fine $\lim u(b)=\lambda \Rightarrow$ semifine $\lim u(b)=\lambda \Leftrightarrow u$ has parabolic limit $\lambda$ at $b$.

Proof. The second part of the theorem is a direct consequence of the first part.

To prove the first part, consider $\lambda<\infty$. Let $\left\{\left(y_{m}, t_{m}\right)\right\}$ be a sequence of points in a parabolic region $P(b ; \alpha)$ converging to $b$ such that for all $\delta>0$ there exists 
$M(\delta) \in \mathbf{N}$ such that $\lambda-\delta<u\left(y_{m}, t_{m}\right)<\lambda+\delta$, if $m \geqslant M(\delta)$. For each $m \in \mathbf{N}$ and $\rho>1$, define,

$$
E_{m, \rho}=\left\{\left(x, \rho t_{m}\right):\left\|x-y_{m}\right\|^{2} \leqslant(\rho-1)^{2} t_{m}\right\} .
$$

Now, since $\theta(\rho) \rightarrow 1$ as $\rho \rightarrow 1$, for any $\delta>0$ there exists $\rho>1$ such that $\lambda-2 \delta<$ $(\lambda-\delta) \theta(\rho)$. Then from Proposition 6.1(i), $u>\lambda-2 \delta$ on the set $E=$ $\cup_{m \geqslant M(\delta)} E_{m, \rho}$. Since by Proposition $3.1, E$ is not semithin at $b$, it follows that $\lambda \leqslant$ semifine lim sup $u(b)$.

For the first inequality, one proceeds in a similar manner. For each $m \in \mathbf{N}$ and $0<\rho<1$, define,

$$
F_{m, \rho}=\left\{\left(x, \rho t_{m}\right):\left\|x-y_{m}\right\|^{2} \leqslant \rho^{-1}(1-\rho)^{2} t_{m}\right\} .
$$

Then $u(x, t) \leqslant \phi(\rho) u\left(y_{m}, t_{m}\right)$ if $(x, t) \in F_{m, \rho}$, which implies that for any $\delta>0$, there exists $0<\rho<1$ such that $u<\lambda+2 \delta$ on a set which is not semithin at $b$.

To complete the proof, consider $\lambda=\infty$. Then only the second inequality needs to be examined. Fix $\rho>1$ and let $\left\{\left(y_{m}, t_{m}\right)\right\}$ be a sequence of points in a parabolic region $P(b ; \alpha)$ converging to $b$ such that $u\left(y_{m}, t_{m}\right)$ tends to $\infty$. Then for any $\delta>0$ there exists $M(\delta) \in \mathbf{N}$ such that $u\left(y_{m}, t_{m}\right)>\delta \theta(\rho)^{-1}$ if $m \geqslant M(\delta)$. Hence, by Propositions 6.1(i) and 3.1, $u>\delta$ on a set which is not semithin at $b$.

REMARK 6.3. This theorem shows that Doob's result (cf. [11, Theorem 5.2]), in the case $h=1$, can be deduced directly from fine convergence without having to use a reduction theorem (cf. Theorem 4.1; [22, Theorem 4.2]).

7. A local fine limit theorem. If $X$ is a Brelot space (satisfying the Axiom of Domination) and $W$ is an open, connected subset of $X$, Koranyi and Taylor [21] obtained a Borel isomorphism between a subset of the Martin boundary of $X$ and a subset of the Martin boundary of $W$. This Borel isomorphism preserves the null sets for the representing measure of a harmonic function on $X$ and the representing measure of its restriction to $W$. This relationship was used in [6 and 22] to obtain local Fatou theorems from the fine limit theorem.

In [21] it was asked whether a similar result holds for strong harmonic spaces. This section presents an affirmative answer to this question for "regular" subsets of strong harmonic spaces.

In this section let $(Y, \mathcal{H})$ be a strong harmonic space, $W$ an open subset of $Y$, and $r$ a reference measure (i.e. $r$ is a regular Borel measure such that $Y$ is the smallest absorbing set containing the support of $r$ ) on $Y$ such that the following conditions are satisfied.

(*) $r(Y)<\infty$ and the restriction of $r$ to $W$ (denoted by $s$ ) is a reference measure on $W$.

(**) $R_{Y \backslash W} u$ is continuous on $Y$ for every nonnegative harmonic $u$ on $Y$.

Clearly $(* *)$ holds if every boundary point of $W$ is regular.

The following notations will be used.

$\mathcal{H}_{+}(Y)$ is the set of all nonnegative harmonic functions on $Y$ endowed with the topology of uniform convergence on compact subsets of $Y$. 
$\mathcal{H}_{r}(Y)=\left\{u \in \mathcal{H}_{+}(Y): \int u d r<\infty\right\}$

$\mathcal{H}_{r}^{1}(Y)=\left\{u \in \mathcal{H}_{+}(Y) ; \int u d r \leqslant 1\right\}$.

$\Delta(Y)=\left\{u \in \mathcal{H}_{+}(Y): u \neq 0, u\right.$ is a minimal harmonic function on $\left.Y\right\}$;

$B_{r}(Y)=\left\{u \in \Delta(Y): \int u d r=1\right\}$.

$\mathfrak{T}_{r}(Y)$ is the set of all finite Borel measures on $\mathcal{H}_{r}^{1}(Y)$ which are supported by $\Delta(Y) \cap \mathcal{H}_{r}^{1}(Y)$.

Observe that measures in $\mathfrak{K}_{r}(Y)$ are regular since $\mathcal{H}_{r}^{1}(Y)$ is a compact metrisable space. By using the Choquet-Meyer existence and uniqueness theorem, Janssen [18, 2.5] obtained the following integral representation theorem.

THEOREM 7.1. For every $u \in \mathscr{H}_{r}(Y)$ there exists a unique $\mu \in \mathfrak{N}_{r}(Y)$ such that $u(x)=\int h(x) d \mu(h)$ for all $x \in Y$. ( $\mu$ is called the representing measure for $u$.)

Conversely, if $\mu \in \mathscr{N}_{r}(Y)$ and $u(x)=\int h(x) d \mu(h)$ for all $x \in Y$, then $u \in \mathcal{K}_{r}(Y)$.

Now, by letting " $\mathcal{S}$ " (resp. " $\odot$ ") in [26] be the set of nonnegative, $r$-integrable superharmonic functions (resp. $r$-integrable potentials) on $Y$, it follows from axiomatic potential theory that all the hypotheses in [26] are satisfied except for (9) and (10), which assume Theorem 7.1 with $\Re_{r}(Y)$ replaced by the space of positive Borel measures on $\Delta(Y) \cap \mathcal{H}_{r}^{1}(Y)$. However, this does not alter the results in [26]. Hence one obtains the following version of the Fatou-Naim-Doob theorem, by means of elementary measure and lattice theory (cf. [18, p. 118]).

THEOREM 7.2. If $u$ and $v$ are positive, r-integrable, harmonic functions on $Y$, then $u / v$ has fine limit $d \mu / d \nu \nu$-a.e. on $B_{r}(Y)$, where $\mu$ and $\nu$ are the representing measures for $u$ and $v$, respectively.

Now, if $Y$ is a Brelot space, then Dirac measures are reference measures and for any $A \subset Y$, and nonnegative superharmonic $u$ on $Y,\left\{x \in A: \hat{R}_{A} u(x)<u(x)\right\}$ is a polar set. This does not hold for parabolic potential theory. However, hypotheses $(*),(* *)$ allow one to apply the methods used in the appendix of [21] to the case of strong harmonic spaces.

Set

$$
\begin{aligned}
B_{r}(Y, W) & =\left\{h \in B_{r}(Y): Y \backslash W \text { is thin at } h\right\} \\
& =\left\{h \in B_{r}(Y): R_{Y \backslash W} h \text { is a potential }\right\} .
\end{aligned}
$$

Proposition 7.3 (CF. [14, Theorem 1; 21, Theorem A.1]). (i) For each $h \in$ $B_{r}(Y, W), h-R_{Y \backslash W} h \in \Delta(W)$.

(ii) If $h, k \in B_{r}(Y, W)$ are such that $h-R_{Y \backslash W} h$ and $k-R_{Y \backslash W} k$ are proportional on $W$, then $h=k$.

Proof. To prove (i) let $h \in B_{r}(Y, W)$ and $w \in \mathcal{H}_{+}(W)$ be such that $w \leqslant h-$ $R_{Y \backslash W} h$ on $W$. Define

$$
v(x)= \begin{cases}w(x)+R_{Y \backslash W} h(x), & \text { if } x \in W, \\ h(x), & \text { if } x \notin W .\end{cases}
$$

Then, by using $(* *)$ it follows that $v$ is a continuous superharmonic function on $Y$, which is harmonic on $W$. Since $v \geqslant 0, v=u+p$ where $u \in \mathscr{H}_{+}(Y)$ and $p$ is a 
continuous potential on $Y$ which is harmonic on $W$. Now, $0 \leqslant v \leqslant h$, hence $0 \leqslant u \leqslant h$ and then the minimality of $h, u=\alpha h$ for some $0 \leqslant \alpha \leqslant 1$. Also the properties of $p$ and the minimum principle in [3, Korollar 2.4.3] imply $R_{Y \backslash W} p=p$. Therefore, by the additivity of the reduced function,

$$
R_{Y \backslash W} h=R_{Y \backslash W} v=R_{Y \backslash W} u+R_{Y \backslash W} p=\alpha R_{Y \backslash W} h+p,
$$

so $p=(1-\alpha) R_{Y \backslash W} h$ on $Y$. Hence,

$$
w=u+p-R_{Y \backslash W} h=\alpha\left(h-R_{Y \backslash W} h\right) \text { on } W .
$$

(ii) is a consequence of the uniqueness of the Riesz decomposition.

LEMMA 7.4.

$$
B_{r}(Y, W)=\left\{h \in B_{r}(Y): \int R_{Y \backslash W} h d r<1\right\}
$$

ProOF.

$$
\int R_{Y \backslash W} h d r=1 \quad \text { iff } \quad h-R_{Y \backslash W} h=0 \quad r \text {-a.e. }
$$

This holds iff $E=\left\{x \in W: h(x)=R_{Y \backslash W} h(x)\right\}$ contains the support of $s$ iff $E=W$, by using $(*)$. The result follows.

Now, for each $p \in \mathbf{N}$ set

$$
B_{r}(Y, W, p)=\left\{h \in B_{r}(Y): \int R_{Y \backslash W} h d r \leqslant 1-\frac{1}{p}\right\} .
$$

Then $B_{r}(Y, W)=\cup_{p=1}^{\infty} B_{r}(Y, W, p)$ and, since the map $h \in \mathcal{K}_{+}(Y) \rightarrow \int R_{Y \backslash W} h d r$ is lower semicontinuous, $B_{r}(Y, W)$ is a Borel set.

Definition 7.5. Define the map $\psi: B_{r}(Y, W) \rightarrow B_{s}(W)$ by

$$
\psi(h)=c(h)\left(h-R_{Y \backslash W} h\right),
$$

where

$$
c(h)^{-1}=\int\left(h-R_{Y \backslash W} h\right) d s=1-\int R_{Y \backslash W} h d r .
$$

The proof of the next result is similar to that of Proposition A.2 in [21] except that results on uniform integrability (cf. [9]) are needed in this case.

Proposition 7.6. $\psi$ is a continuous injective mapping on each $B_{r}(Y, W, \rho)$.

Proof. $\psi$ is injective on $B_{r}(Y, W)$ by Proposition 7.3. Let $\left\{h_{m}\right\}$ converge to $h$ in $B_{r}(Y, W, p)$. Since $\mathcal{H}_{s}^{1}(W)$ is compact, there exists $u \in \mathcal{H}_{s}^{1}(W)$ and a subsequence $\left\{h_{i(m)}\right\}$ such that $\left\{h_{i(m)}-R_{Y \backslash W} h_{i(m)}\right\}$ converges to $u$ in $\mathcal{H}_{s}^{1}(W)$. Then, for all $x \in W$,

$$
u(x)=h(x)-\lim _{m \rightarrow \infty} R_{Y \backslash W} h_{i(m)}(x) \leqslant h(x)-R_{Y \backslash W} h(x) .
$$

Hence $u=\alpha\left(h-R_{Y \backslash W} h\right)$ on $W$ for some $0 \leqslant \alpha \leqslant 1$. Now, $\left\{h_{i(m)}\right\} \subset L^{1}(r)$ converges pointwise to $h$, and for all $m, \int h_{i(m)} d r=1=\int h d r$. Therefore, $\left\{h_{i(m)}\right\}$ is uniformly integrable with respect to $r$ (cf. [9, Theorem 4.5.4]). Since $0 \leqslant h_{i(m)}-$ $R_{Y \backslash W} h_{i(m)} \leqslant h_{i(m)}$, it follows that $\left\{h_{i(m)}-R_{Y \backslash W} h_{i(m)}\right\}$ is uniformly integrable with 
respect to $s$. Therefore, by another application of Theorem 4.5.4 in [9],

$$
\lim _{m \rightarrow \infty} \int h_{i(m)}-R_{Y \backslash W} h_{i(m)} d s=\int u d s .
$$

Consequently, $\alpha>0$ and $\lim _{m \rightarrow \infty} c\left(h_{i(m)}\right)=\alpha^{-1} c(h)$. Hence, $\left\{\psi\left(h_{i(m)}\right)\right\}$ converges to $\psi(h)$.

The proofs of the next two results are the same as those of Corollary A.3 and Proposition A.5 in [21].

Corollary 7.7. If $E \subset B_{r}(Y, W)$ is Borel then $\psi(E)$ is Borel.

THEOREM 7.8. Let $v \in \mathcal{H}_{r}(Y), \nu$ be the representing measure for $v$, and $\omega$ be the representing measure for $\left.v\right|_{W}$. Then for any Borel set $E \subset B_{r}(Y, W)$,

$$
\omega(\psi(E))=\int_{E} c(h)^{-1} d \nu(h) .
$$

Hence, $\nu(E)=0 \Leftrightarrow \omega(\psi(E))=0$.

The following result is a restatement of Exercise 5.3.1 in [10] (cf. [21, Lemma A.7]).

LEMMA 7.9. Let $u$ be a nonnegative superharmonic function on $Y$ and $A \subset W$. Then,

$$
\left(R^{W}\right)_{A}\left(u-R_{Y \backslash W} u\right)=R_{A \cup(Y \backslash W)} u-R_{Y \backslash W} u \text { on } W,
$$

where $R^{W}$ is the réduite operator with respect to the space $W$.

For any $h \in B_{r}(Y)$, the fine filter (on $Y$ ) at $h$ will be denoted by $\mathscr{F}(h)$ and its restriction to $W$ by $\left.\mathscr{F}(h)\right|_{W}$.

For any $k \in B_{s}(W)$, the fine filter (on $W$ ) at $k$ will be denoted by $\mathscr{F}_{W}(k)$.

Then, by using Lemma $2.12,\left.\mathscr{F}(h)\right|_{W}=\mathscr{F}_{W}(\psi(h))$ for all $h \in B_{r}(Y, W)$.

Consequently, the main result of this section follows from Theorem 7.2 applied to $W$ and Theorem 7.8 .

THEOREM 7.10. Let $u>0$ be s-integrable, harmonic on $W$, and $v>0$ be r-integrable, harmonic on $Y$ with representing measure $\nu$. Then the limit of $u / v$ along $\left.\mathscr{F}(h)\right|_{W}$ is $d \mu / d \omega(\psi(h))$ for $\nu$-a.e. $h \in B_{r}(Y, W)$, where $\mu, \omega$ are the representing measures for $u$, $\left.v\right|_{W}$, respectively.

8. A reduction theorem. In this section let $\mathcal{H}$ be a set of nonnegative functions on a set $Z$ such that:

(i) $\mathcal{H}+\mathcal{H} \subset \mathcal{H}$;

(ii) $\mathcal{H}$ contains constants;

(iii) $f, g \in \mathcal{H}, g \geqslant f \Rightarrow g-f \in \mathcal{H}$;

(iv) $\mathcal{H}$ is a lattice with respect to the usual ordering of functions (denote lattice operations by $\wedge, \vee)$.

Let $(B, \Re, \nu)$ be a measure space such that for each $b \in B$ there exist two filters $\mathcal{G}_{1}(b)$ and $\mathcal{G}_{2}(b)$ on $Z$. For any $f \in \mathcal{H}, b \in B$ and $i \in\{1,2\}$, let $\mathcal{S}_{i}$-limsup $f(b)$ (resp. $\left.\mathcal{G}_{i}-\lim \inf f(b)\right)$ denote the $\lim \sup \left(\right.$ resp. $\lim \inf$ ) of $f$ along $\mathcal{G}_{i}(b) . \mathcal{G}_{i}-\lim f(b)$ denotes the limit of $f$ along $\mathcal{G}_{i}(b)$ if it exists. Further, assume the following conditions are satisfied. 
(v) For all $f \in \mathscr{H}, \mathcal{G}_{1}-\lim f(b)=l(f, b)$ is finite for $\nu$-a.e. $b \in B$.

(vi) For each $f, g \in \mathcal{H}, l(f \wedge g, b)=\min \{l(f, b), l(g, b)\}$ and $l(f \vee g, b)=$ $\max \{l(f, b), l(g, b)\}$ if $l(f, b)$ and $l(g, b)$ exist.

THEOREM 8.1. Assume for each $f \in \mathcal{H}, \mathcal{G}_{1}-\lim f(b)=0 \Rightarrow \mathcal{G}_{2}-\lim f(b)=0$ for v-a.e. $b \in B$. Then, for all $f \in \mathcal{H}, \mathcal{G}_{2}-\lim f(b)=l(f, b)$ for v-a.e. $b \in B$.

Proof. Fix $f \in \mathcal{H}$. For any positive rational number $p$ and integer $n \geqslant 0$, let $E_{p, n}=\{b \in B: n p \leqslant l(f, b) \leqslant(n+1) p\}$ and $E_{p}=\cup_{n=0}^{\infty} E_{p, n}$. Then for each $p$, $\nu\left(B \backslash E_{p}\right)=0$ (by condition (v)). Now, for any $p$ and $n$, the conditions on $\mathcal{H}$ imply $n p-f \wedge n p$ and $f \vee(n+1) p-(n+1) p$ are in $\mathfrak{K}$. Hence, for any $b \in E_{p, n}$, condition (vi) implies

$$
l(n p-f \wedge n p, b)=0=l(f \vee(n+1) p-(n+1) p, b) .
$$

Then, by the hypothesis, there exists a set $F_{p, n} \subset E_{p, n}$ such that $\nu\left(F_{p, n}\right)=0$ and, for all $b \in E_{p, n} \backslash F_{p, n}$,

$$
\mathcal{G}_{2}-\lim (f \wedge n p)(b)=n p, \quad \mathcal{G}_{2}-\lim (f \vee(n+1) p)(b)=(n+1) p .
$$

Now, $f \wedge n p \leqslant f \leqslant f \vee(n+1) p$. Hence, for all $b \in E_{p, n} \backslash F_{p, n}$,

$$
n p \leqslant \mathcal{G}_{2}-\liminf f(b) \leqslant \mathcal{G}_{2}-\lim \sup f(b) \leqslant(n+1) p,
$$

which implies

$$
\left|l(f, b)-\left(\mathcal{G}_{2}-\liminf f(b)\right)\right| \leqslant p, \quad\left|l(f, b)-\left(\mathcal{G}_{2}-\lim \sup f(b)\right)\right| \leqslant p .
$$

Hence, $\mathcal{G}_{2}-\lim f(b)=l(f, b)$ for all $b \in E=\cap_{p}\left(E_{p} \backslash \cup_{n=0}^{\infty} F_{p, n}\right)$, and it is clear that $\nu(B \backslash E)=0$.

This result was proved in [22, Theorem 1.2] in case $h>0$ is harmonic on a space $Z ; \mathcal{H}=\{u / h: u \geqslant 0$ is harmonic on $Z\} ; B$ parameterises the minimal harmonic functions on $Z$; every nonnegative harmonic function $u$ on $Z$ is uniquely represented by a measure $\mu_{u}$ on $(B, \mathfrak{T})$ in terms of minimal harmonic functions; and

$$
\mathcal{G}_{1}-\lim \frac{u}{h}(b)=\frac{d \mu_{u}}{d \mu_{h}}(b) \text { for } \mu_{h} \text {-a.e. } b \in B .
$$

9. A local Fatou theorem. Let $L$ be the second-order parabolic differential operator defined in $\S 1$. This section establishes a Carleson-type local Fatou theorem for solutions of $L u=0$ on a union of parabolic regions.

Lemma 9.1 (CF. [6, ThEorème 8]). Let $E \subset \mathbf{R}^{n}$ and $W \subset X$ be such that for each $b \in E, W$ contains a parabolic region with vertex $b$. Then, for a.e. $b \in E, W$ contains parabolic regions of arbitrary aperature with vertex $b$.

Proof. It suffices to assume that for each $b \in E, W$ contains $P(b ; \alpha, \delta)$ for fixed $\alpha, \delta$. Choose $m_{0} \in \mathbf{N}$ such that $1 / m_{0}<\delta$. Let $\beta>0$ and for each $m \geqslant m_{0}$, define $E_{m}=\{b \in E: P(b ; \beta, 1 / m) \subset W\}$. Let $D$ denote the set of points of strong density of $E$ (cf. [7, p. 49]). It suffices to prove that $D \subset \cup_{m \geqslant m_{0}} E_{m}$. If $b \notin E_{m}$ for all $m \geqslant m_{0}$ there exists a sequence of points $\left\{\left(x_{m}, t_{m}\right): m \geqslant m_{0}\right\} \subset P(b ; \beta, 1 / m) \backslash W$. For each $m \geqslant m_{0}$, define $F_{m}=\left\{y \in \mathbf{R}^{n}:\left\|y-x_{m}\right\|^{2}<\alpha t_{m}\right\}$. Then $y \in F_{m}$ implies $\left(x_{m}, t_{m}\right) \in P(y ; \alpha, 1 / m)$. Hence $F_{m} \cap E=\varnothing$ for all $m \geqslant m_{0}$. Now, the ball $F_{m}$ has 
radius $\left(\alpha t_{m}\right)^{1 / 2}$ and is contained in the ball of centre $b$ and radius $\left(\alpha^{1 / 2}+\gamma^{1 / 2}\right) t_{m}^{1 / 2}$. Since $t_{m} \rightarrow 0$, this implies $b \notin D$.

The main result of this section will now be proved.

THEOREM 9.2. Let $E \subset \mathbf{R}^{n}$ and $W$ be an open subset of $X$ which contains a parabolic region $W_{b}$ with vertex $b$ for each $b \in E$. Let $u$ be a harmonic function on $W$ which is either upper or lower bounded on $W_{b}$ for each $b \in E$. Then $u$ has finite parabolic limits a.e. on $E$.

Proof. It suffices to assume $E$ is compact, $W_{b}=P(b ; \alpha, \delta)$ for all $b \in E$, where $\alpha, \delta$ are fixed, and $W=\cup_{b \in E} W_{b}$. Then, by constructing a barrier (cf. [12, Chapter $3, \S 4]$ ), the boundedness of the coefficients of $L$ implies every boundary point of $W$ in $\mathbf{R}^{n} \times(0, \delta)$ is a regular boundary point of $W$. This implies that for every harmonic $u \geqslant 0$ on $X, R_{X \backslash W} u$ is continuous on the intersection of the boundary of $W$ with $X \cap\{(x, t): t<\delta\}$. However, the continuity of $R_{X \backslash W} u$ at points on $t=\delta$ does not follow. This difficulty can be avoided by considered the space $X \cap\{(x, t)$ : $t<\delta\}$ instead of $X$. That is, it suffices to assume $T=\delta$. Then condition (**) in $\S 7$ is satisfied for the harmonic space $X$. Furthermore, by considering the sets $\{b \in E$ : $u>-m$ on $\left.W_{b}\right\}$ and $\left\{b \in E: u<m\right.$ on $\left.W_{b}\right\}$ for each $m \in \mathbf{N}$, one can assume $u>1$ on $W$. This condition will be useful in choosing a suitable reference measure.

Now, let $\tau$ be a finite reference measure on $W$ and fix a point $(y, \eta) \in X$ such that $y$ is outside the projection of $W$ on $\mathbf{R}^{n}$.

Define the measure $r$ on $X$ by

$$
\int f d r=\int_{\eta}^{\delta} t^{(n-4) / 2} f(y, t) d t+\int \frac{f}{u} d \tau
$$

for every nonnegative Borel function $f$ on $X$. Then $\int f d s=\int f / u d \tau$ for every nonnegative Borel $f$ on $W$. Hence $s$ is a reference measure on $W$. The strong maximum principle of Nirenburg (cf. [24, Theorem 4]) implies that $r$ is a reference measure. The conditions on $u$ imply $r(X), s(W)$ and $\int u d s$ are finite. Hence condition $(*)$ in $\$ 7$ is satisfied.

It will now be shown that $B_{r}(X)$ can be identified with $\mathbf{R}^{n}$. For each $b \in \mathbf{R}^{n}$, define $Q(b)=\int K_{b} d r$. Now, for each $b \in \mathbf{R}^{n}, K_{b}$ is a positive continuous function on $X$, hence $0<Q(b)<\infty$. Furthermore, $K_{b}(x, t) \leqslant C t^{-n / 2}$ for all $(x, t) \in X$ and $t^{-n / 2}$ is $r$-integrable, hence $Q$ is a continuous function on $\mathbf{R}^{n}$.

Define the map $\Omega: \mathbf{R}^{n} \rightarrow B_{r}(X)$ by $\Omega(b)=Q(b)^{-1} K_{b}$.

Now, let $\left\{b_{m}\right\}$ be a sequence in $\mathbf{R}^{n}$ converging to $b \in \mathbf{R}^{n}$. Then it is well known that $K_{b_{m}} \rightarrow K_{b}$ pointwise. However, it can also be shown that $K_{b_{m}} \rightarrow K_{b}$ uniformly on compact subsets of $X$ (see Remark 9.3 below). This implies $\Omega$ is continuous, and it is clearly bijective. It remains to show that $\Omega^{-1}$ is continuous. To do this, a lower bound for $Q(b)$ will be needed. For any $b \in \mathbf{R}^{n}$,

$$
\begin{aligned}
\int K_{b} d r & \geqslant \int_{\eta}^{\delta} t^{(n-4) / 2} K_{b}(y, t) d t \geqslant C \int_{\eta}^{\delta} t^{-2} \exp \left(-\frac{\|y-b\|^{2}}{4 p_{1} t}\right) d t \\
& =C\|y-b\|^{-2}\left\{1-\exp \left[\frac{1}{4 p_{1}}\left(\frac{1}{\delta}-\frac{1}{\eta}\right)\|y-b\|^{2}\right]\right\} \exp \left(-\frac{\|y-b\|^{2}}{4 p_{1} \delta}\right) .
\end{aligned}
$$


Hence, for all $(x, t) \in Y$,

$$
\begin{aligned}
\Omega(b)(x, t) \leqslant & C t^{-n / 2}\|y-b\|^{2} \exp \left(\frac{\|y-b\|^{2}}{4 p_{1} \delta}-\frac{\|x-b\|^{2}}{4 p_{2} t}\right) \\
& \times\left\{1-\exp \left[\frac{1}{4 p_{1}}\left(\frac{1}{\delta}-\frac{1}{\eta}\right)\|y-b\|^{2}\right]\right\}^{-1} .
\end{aligned}
$$

Therefore, if a sequence $\left\{b_{m}\right\} \subset \mathbf{R}^{n}$ approaches $\infty, \Omega\left(b_{m}\right)(x, t) \rightarrow 0$ for all $t<$ $\min \left(p_{1} p_{2}^{-1} \delta, \delta\right)$ and $x \in \mathbf{R}^{n-1} \times \mathbf{R}_{+}$. So the sequence $\left\{\Omega\left(b_{m}\right)\right\}$ is not convergent in $B_{r}(X)$. Hence, if $\left\{\Omega\left(b_{m}\right)\right\}$ converges to $\Omega(b)$ in $B_{r}(X)$, the sequence $\left\{b_{m}\right\}$ must be bounded. Since $\Omega$ is continuous and injective, every limit point of $\left\{b_{m}\right\}$ is $b$. Consequently, $\Omega^{-1}$ is continuous and $\mathbf{R}^{n}$ is homeomorphic to $B_{r}(X)$.

Property (P5) in $\$ 1$ implies $Q(b)^{-1} d b$ is the representing measure for the constant function 1 on $X$. Now, from Lemmas 5.1 and 9.1, there is a set $E_{1} \subset E$ such that:

(i) $E \backslash E_{1}$ is of Lebesgue measure zero;

(ii) $X \backslash W$ is thin at every $b \in E_{1}$;

(iii) for each $b \in E_{1}, W$ contains parabolic regions of arbitrary aperature with vertex $b$.

Condition (ii) implies $E_{1} \subset B_{r}(X, W)$, so $E_{1}$ is mapped injectively onto the subset $\psi\left(E_{1}\right)$ of $B_{s}(W)$ by the map $\psi$ defined in $\S 7$. Now, let $Z=W, \mathcal{H}=\mathcal{H}_{s}(W)$ and $B=E_{1}$ in $\S 8$. Since $\mathcal{H}_{+}(W)$ satisfies conditions (i)-(iv) in $\S 8$, it is easy to deduce that $\mathcal{H}_{s}(W)$ also satisfies these conditions. For each $b \in B$ and $f \in \mathcal{H}$, let $l(f, b)=$ $d \mu(\psi(b)) / d \omega$, where $\mu$ (resp. $\omega$ ) is the representing measure for $f$ (resp. 1) on $B_{s}(W)$. For each $b \in B$, let $\mathcal{G}_{1}(b)=\left.\mathscr{F}(b)\right|_{W}$ and $\mathcal{G}_{2}(b)=\left.\mathscr{P}(b)\right|_{W}$. Since, $E_{1} \subset$ $B_{r}(X, W)$, Theorem 7.10 implies, for all $f \in \mathcal{H}, \mathcal{G}_{1}-\lim f(b)=l(f, b)$ for Lebesgue a.e. $b \in B$. Now, it follows from measure theory that $l(f, b)$ satisfies condition (vi) in $\$ 8$ (cf. [26, p. 160]). Now, since $E_{1}$ satisfies condition (iii) above, Theorem 4.1 implies, for all $f \in \mathcal{H}$ and $b \in B, \mathcal{G}_{1}-\lim f(b)=0 \Rightarrow \mathcal{G}_{2}-\lim f(b)=0$. The result follows from Theorem 8.1.

REMARK 9.3. Let $\left\{b_{m}\right\}$ be a sequence in $\mathbf{R}^{n}$ converging to $b \in \mathbf{R}^{n}$. The literature does not seem to explicitly state that $K_{b_{m}} \rightarrow K_{b}$ uniformly on compact subsets of $X$. A proof of this is now given under the assumptions made in the proof of the above theorem.

Let $\left\{b_{m}\right\}$ be as above. Now, it is well known that $W_{2}\left(x, t ; b_{m}, 0\right) \rightarrow W_{2}(x, t ; b, 0)$ uniformly on compact subsets of $\mathbf{R}^{n} \times \mathbf{R}_{+}$, hence

$$
\int W_{2}\left(x, t ; b_{m}, 0\right) d r(x, t) \rightarrow \int W_{2}(x, t ; b, 0) d r(x, t)
$$

Therefore the sequence $\left\{W_{2}\left(\cdot, \cdot ; b_{m}, 0\right)\right\}$ of functions is uniformly integrable with respect to $r$. Since $0 \leqslant \Gamma \leqslant P W_{2}$, the sequence $\left\{K_{b_{m}}\right\}$ of functions is uniformly integrable with respect to $r$. Hence $\int K_{b_{m}} d r \rightarrow \int K_{b} d r$. Recall that $\mathcal{H}_{r}^{1}(X)$ is compact (with respect to the topology of uniform convergence on compact subsets of $X$ ). The result follows by considering subsequences of $\left\{\left[j K_{b_{m}} d r\right]^{-1} K_{b_{m}}\right\}$. 


\section{REFERENCES}

1. D. G. Aronson, Bounds for the fundamental solution of a parabolic equation, Bull. Amer. Math. Soc. 73 (1967), 890-896.

2. Non-negative solutions of linear parabolic equations, Ann. Scuola Norm. Sup. Pisa 22 (1968), 607-694.

3. H. Bauer, Harmonische Räume und ihre Potentialtheorie, Lecture Notes in Math., vol. 22, SpringerVerlag, New York and Berlin, 1966.

4. N. Bourbaki, General topology, Part 2, Addison-Wesley, Reading, Mass., 1966.

5. M. Brelot, On topologies and boundaries in potential theory, Lecture Notes in Math., vol. 175, Springer-Verlag, 1971.

6. M. Brelot and J. L. Doob, Limites angulaires et limites fines, Ann. Inst. Fourier (Grenoble) 13 (1963), 395-415.

7. A. P. Calderon, On the behaviour of harmonic functions at the boundary, Trans. Amer. Math. Soc. 68 (1950), 47-54.

8. L. Carleson, On the existence of boundary values for harmonic functions in several variables, Ark. Mat. 4 (1961), 393-399.

9. Kai Lai Chung, $A$ course in probability theory, 2nd ed., Academic Press, New York, 1974.

10. C. Constantinescu and A. Cornea, Potential theory on harmonic spaces, Springer-Verlag, New York and Berlin, 1972.

11. J. L. Doob, Relative limit theorems in analysis, J. Analyse Math. 8 (1960/61), 289-306.

12. A. Friedman, Partial differential equations of parabolic type, Prentice-Hall, Englewood Cliffs, N. J., 1964.

13. K. Gowrisankaran, Extreme harmonic functions and boundary value problems, Ann. Inst. Fourier (Grenoble) 13 (1963), 307-356.

14. __ Extreme harmonic functions and boundary value problems. II, Math. Z. 94 (1966), 256-270.

15. S. Guber, On the potential theory of linear homogeneous parabolic partial differential equations of second order, Lecture Notes in Math., vol. 31, Springer-Verlag, 1967, pp. 112-117.

16. J. R. Hattemer, Boundary behaviour of temperatures. I, Studia Math. 25 (1964), 111-155.

17. R. A. Hunt and R. L. Wheeden, Positive harmonic functions on Lipschitz domains, Trans. Amer. Math. Soc. 147 (1970), 507-527.

18. K. Janssen, Martin boundary and $\mathcal{H}^{P}$-theory of harmonic spaces, Lecture Notes in Math., vol. 226, Springer-Verlag, New York and Berlin, 1971, pp. 102-151.

19. R. Johnson, Representation theorems and Fatou theorems for second order linear parabolic partial differential equations, Proc. London Math. Soc. (3) 23 (1971), 325-347.

20. J. T. Kemper, Temperatures in several variables: kernel functions, representations, and parabolic boundary values, Trans. Amer. Math. Soc. 167 (1972), 243-262.

21. A. Koranyi and J. C. Taylor, Fine convergence and admissible convergence for symmetric spaces of rank one, Trans. Amer. Math. Soc. 263 (1981), 169-181. , Fine convergence and parabolic convergence for the Helmholtz equation and the heat equation, Illinois J. Math. 27 (1983), 77-93.

23. L. Naim, Sur le rôle de la frontière de R. S. Martin dans la théorie du potentiel, Ann. Inst. Fourier (Grenoble) 7 (1957), 183-281.

24. L. Nirenberg, A strong maximum principle for parabolic equations, Comm. Pure Appl. Math. 6 (1953), 167-177.

25. D. Resch, Temperature bounds on an infinite rod, Proc. Amer. Math. Soc. 3 (1952), 632-634.

26. J. C. Taylor, An elementary proof of the theorem of Fatou-Naim-Doob, 1980 Seminar on Harmonic Analysis (Montreal, Que., 1980), CMS Conf. Proc., vol. 1, Amer. Math. Soc., Providence, R. I., 1981, pp. 153-163.

Department of Mathematics, University of the West Indies, Mona, Kingston 7, Jamaica, West INDIES 